\title{
Coronary arterial lesions and myocardial necrosis in stillbirths and infants
}

\author{
D. J. DE SA
}

Department of Pathology, McMaster University Medical Centre, Hamilton

SUMMARY In a detailed study of the coronary arterial tree and myocardium in 256 stillbirths and infants, abnormalities of the coronary arterial tree were noticed in 79 infants, and necrotic lesions of the myocardium in 111 infants. Of the 79 infants with arterial lesions, 70 had associated myocardial necrosis or scarring, or both; the group with coronary arterial lesions, therefore, accounted for the majority of cases with myocardial damage.

The myocardial lesions varied from small zones of subendocardial damage, to larger 'geographical' zones of necrosis scattered haphazardly through the myocardium, and a small group where massive necrotic lesions of the papillary muscles were present. While the coronary arterial lesions were associated with all three patterns, they were particularly found in association with the 'geographical' and papillary muscle changes.

The coronary arterial lesions varied from zones of acute focal, medial necrosis to severe proliferative intimal lesions and medial defects, with a distinct progression of changes from the acute to the more established lesions. The coronary arterial lesions were seen most commonly in association with conditions that could produce severe hypoxia, and it is argued that they result from hypoxia. It is suggested further that the myocardial damage, so commonly associated with the coronary arterial lesions, could compromise the ability of the hypoxic infant to respond to such an insult.

The coronary arterial lesions seen in this group of young infants could offer one explanation for the later development of a variety of other pathological conditions seen in adolescents and young adults.

In 1946 Dock described proliferative intimal changes in the coronary arterial tree of infants and young adults, and his findings were confirmed and extended by many others (Fangman and Hellwig, 1947; Minkowski, 1947; Lober, 1953; Levene, 1956; Schornagel, 1956; Moon, 1957; Robertson, 1960; Vlodaver et al., 1967). Gruenwald (1949) reported necrotic lesions in the coronary arteries of 21 stillbirths and infants and he commented on their association with small foci of myocardial necrosis. He was unable, however, to follow their natural history.

Several reports describe myocardial necrosis in newborn babies and infants. Esterly and Oppenheimer (1967), and Franciosi and Blanc (1968) found evidence of myocardial necrosis in

Department of Pathology, McMaster University Medical Centre

D. J. DE SA, associate professor of pathology infants with a variety of congenital malformations of the heart and great vessels. Berry (1967), in a review of cases of myocardial necrosis occurring in infants and young children, reported several infants with myocardial necrosis, one of whom showed evidence of coronary artery occlusion. Gault and Usher (1960) described a neonate with coronary thrombosis and myocardial infarction complicating birth asphyxia. Bucciarelli et al. (1977), in a study of acquired tricuspid insufficiency in the newborn, found large areas of necrosis in the anterior papillary muscle of one such infant, but did not mention any changes in the coronary vessels.

In an earlier paper (de Sa, 1977), a wide spectrum of myocardial lesions was found in a group of immature hypoxic infants whose lives had been sustained by prolonged assisted ventilation. In that report, destructive lesions of the smaller, intramural branches of the coronary arterial tree were noted but were not elaborated on. The present report is 
based on a detailed study of the coronary arterial tree in 256 stillbirths and infants. The presence of nonflammatory, necrotic, and degenerative lesions of the coronary arterial tree is described and their close association with myocardial necrosis and scarring is stressed.

\section{Materials and methods}

The necropsy records of the McMaster University Medical Centre provided most of the case material, but additional material was obtained through the co-operation of colleagues in St Joseph's and Henderson General Hospitals and the forensic pathology unit, Hamilton. The hearts and coronary vessels of 82 fresh stillbirths, 86 first-week neonatal deaths, 33 other neonatal deaths ages $<28$ days, 24 infants requiring prolonged ventilation (de $\mathrm{Sa}$, 1977), 13 cases of sudden infant death syndrome, and 18 other infants age <one year were studied. In most instances the necropsies were conducted personally or the heart was available for a detailed study. Apart from the blocks of myocardium selected for routine histology, samples of myocardium were available in the reserve stocks taken at the time of necropsy. Even in retrospect, therefore, a detailed examination could be made.

Hearts with congenital malformations of the septum, great vessels, or valves were excluded. Multiple blocks of myocardium, including endocardial and epicardial surfaces, were embedded in paraffin and each block was studied at several different levels.

The numbers of blocks studied depended on the size of the heart. However, in all cases, at least 3 blocks were taken through the free wall of both ventricles, and these included the papillary muscles and atrioventricular grooves. In addition, at least 3 blocks were sampled through the entire thickness of the interventricular septum, including both anterior and posterior epicardial surfaces. Separate blocks were taken to include the left and right coronary arteries near their respective aortic ostia. In the larger hearts, further transversely orientated blocks were taken through an area of myocardium bounded posteriorly by the opening of the coronary sinus and extending anteriorly to include membranous interventricular septum and adjacent muscularis for a distance of approximately $0 \cdot 3 \mathrm{~cm}$. These blocks included the atrioventricular node, bundle of His, and both bundle branches. Sections were cut through these blocks at several levels and stained with a variety of standard histological techniques, as appropriate.

In some cases, blocks of formalin-fixed tissue were processed for ultrastructural study after selecting the areas under a Leitz dissecting microscope. These blocks were embedded in epon, and sections were cut at $1 \mu \mathrm{m}$ and stained with toluidine blue. Specific areas were chosen from these blocks and sectioned on an LKB ultramicrotome and stained with lead citrate and uranyl acetate, and examined on a Philips 300 electron microscope.

\section{Results}

Myocardial lesions. In this series, myocardial lesions (including acute necrosis, replacement with granulation tissue and/or scarring) were found in 111, and coronary arterial lesions (to be described below) were found in $79(31 \%)$ of the 256 infants. However of the 79 infants with coronary arterial lesions, $70(89 \%)$ had associated myocardial lesions. Infants with coronary arterial lesions, while only constituting a minority of the infants studied, accounted for most cases (70 of 111 or $63 \%$ ), with histologically detectable myocardial damage.

The myocardial lesions that were identified were similar in their range and severity to those identified as part of the considerably smaller, earlier series (de Sa, 1977). Generally focal band-like areas of necrosis or scarring were present (83 of 111 infants). These lesions were most often seen in the subendocardial areas of the myocardium (Fig. 1) but not exclusively so. Next in frequency (29 of 111 infants) were irregular geographical areas of necrosis or scarring, scattered haphazardly through both ventricles often associated with small collars of surviving myocardium around intramural vessels (Fig. 2). Finally large areas of necrosis or scarring affecting appreciable areas of papillary muscles were identified in 16 of the 111 infants (Fig. 3). As Table 1 shows, several infants had more than one pattern of necrosis. Furthermore, it is clear that while coronary arterial damage was associated with all three patterns of injury, it was more often associated with the larger lesions.

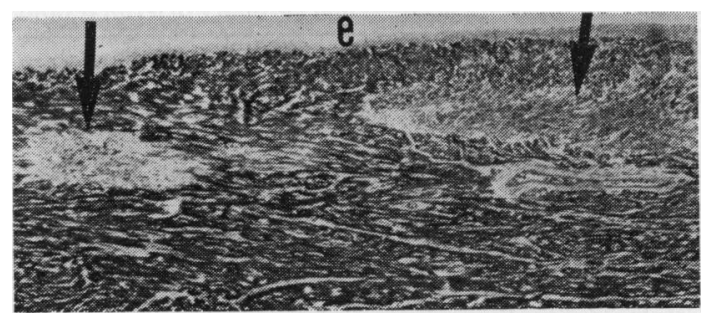

Fig. 1 Small zones of subendocardial fibre necrosis (arrows) are seen in the heart of this 14-day-old infant, 34 weeks' gestation. Lesion on left is somewhat more advanced. Endocardial surface is indicated by ' $e$ '. Gomon's trichrome $(G T) \times 150$. 


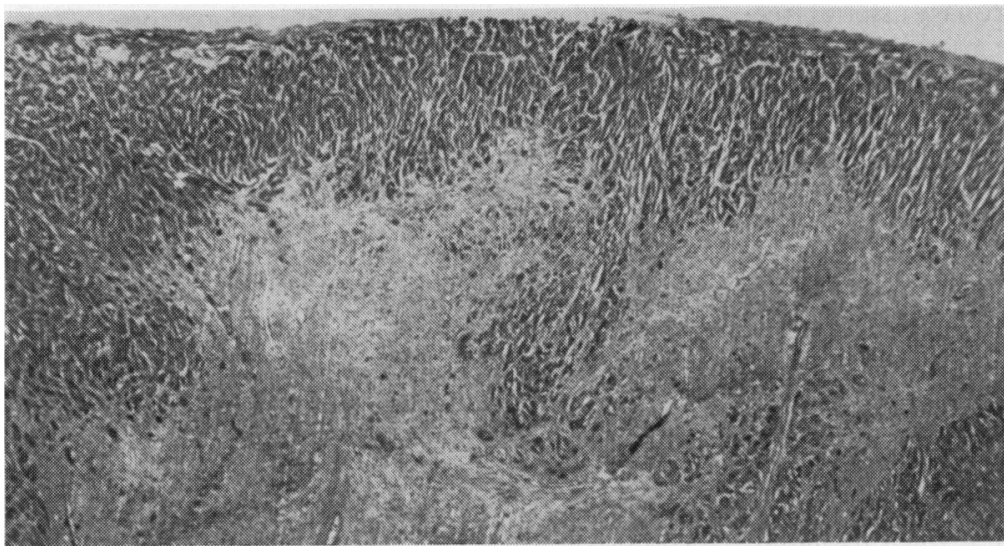

Fig. 2 Large, pale-staining 'geographical' zones of scar tissue, replacing areas of previous myocardial necrosis are seen in this section through the interventricular septum of a 102-day-old infant, maintained on prolonged ventilation. $G T \times 25$.

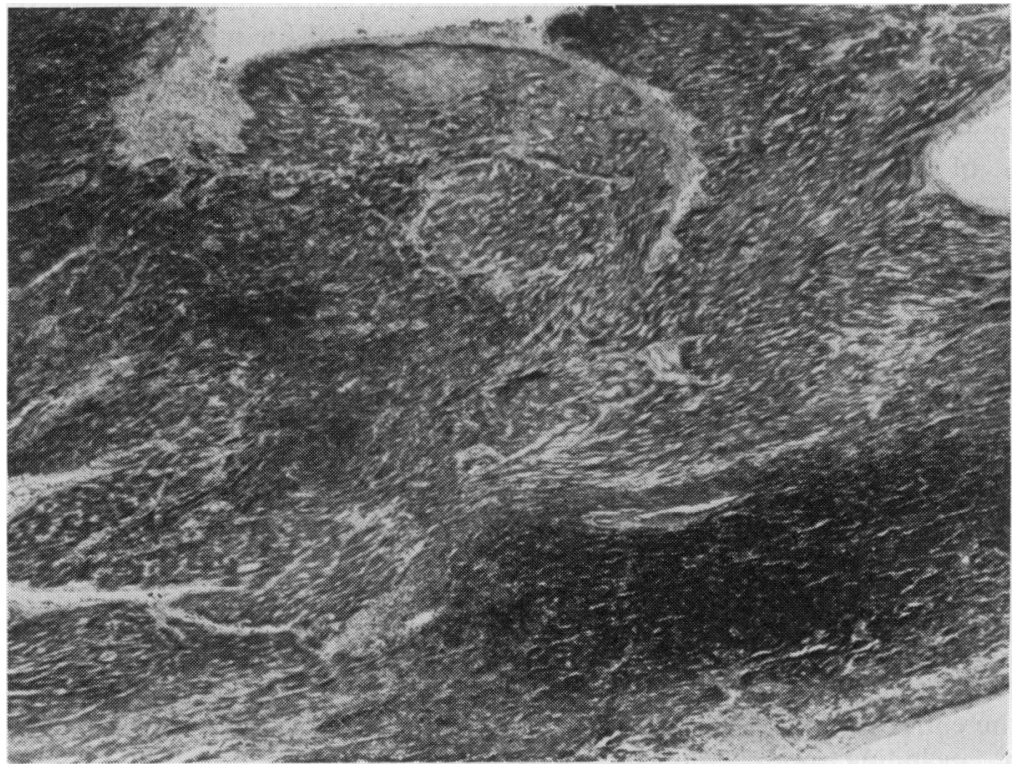

Fig. 3 Section shows massive acute necrosis (darkly stained) occurring in the posterior papillary muscle of the mitral valve of a 5-day-old infant, 29 weeks' gestation, born after a pregnancy complicated by abruptio placentae. GT $\times 180$.

Table 1 Myocardial necrosis and coronary arterial lesions

\begin{tabular}{llll}
\hline $\begin{array}{l}\text { Type of } \\
\text { myocardial lesion }\end{array}$ & $\begin{array}{l}\text { Infants with } \\
\text { coronary arterial } \\
\text { lesions } \\
(n=79)\end{array}$ & $\begin{array}{l}\text { Infants without } \\
\text { coronary arterial } \\
\text { lesions } \\
(n=177)\end{array}$ & $\begin{array}{l}\text { Total no. of } \\
\text { myocardial } \\
\text { lesions } \\
(n=256)\end{array}$ \\
\hline Focal & 48 & 35 & 83 \\
Geographical & 17 & 12 & 29 \\
Papillary muscle & 11 & 5 & 16 \\
None & 9 & 136 & 145 \\
\hline
\end{tabular}

Histological findings in the coronary vessels

The impetus for the present study was provided by a chance observation of a section through an epicardial petechial haemorrhage in a one-day-old infant of 28 weeks' gestation, which showed an area of obvious medial necrosis in the anterior descending coronary artery. In adjacent sections disruption of the internal elastic lamina, early thrombus formation, and imminent rupture of the wall were demonstrated (Fig. 4).

Early necrotic lesions appeared as small areas of smudging of medial cellular detail. They were seen to greatest advantage near areas of perivascular petechial haemorrhages, associated with the rupture of small capillaries in connective tissue around the larger vessels, both on the surface of the heart and intramurally. The lesions were readily seen in $1 \mu \mathrm{m}$ sections (Fig. 5). In many of these cases, step sectioning through the block showed focal micro- 


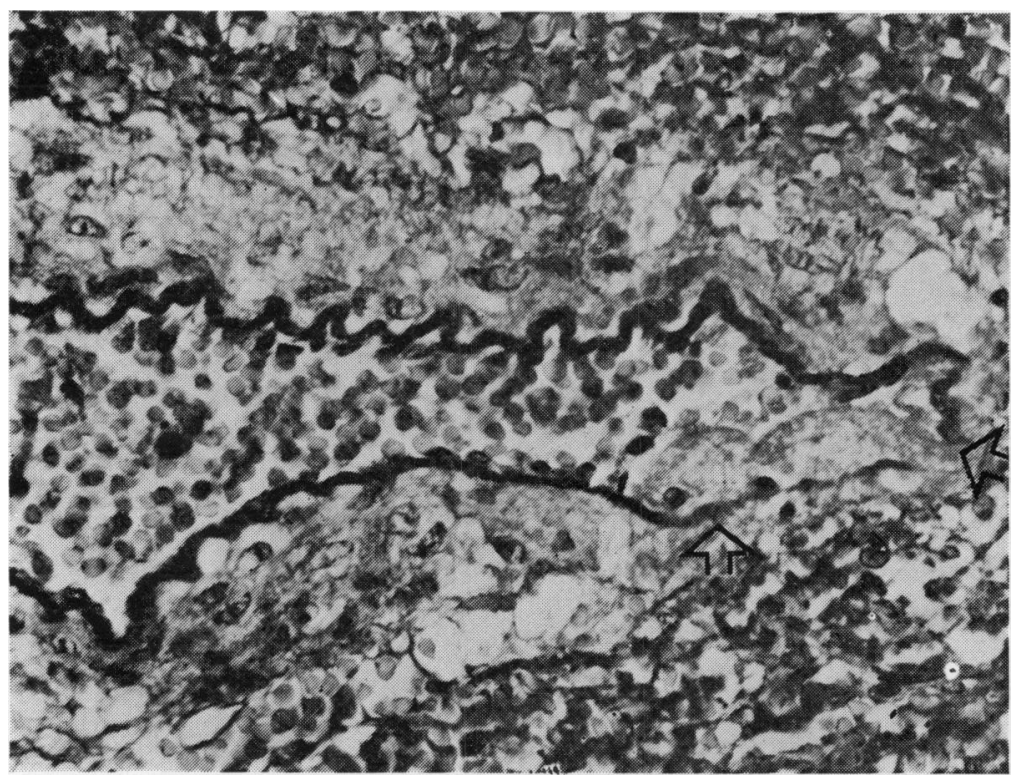

Fig. 4 Disruption of the internal elastic lamina (zone between arrows), is seen in this section, through the anterior descending coronary artery of a 2-day-old infant of 28 weeks' gestation. Note globular, amorphous masses of thrombus present in relation to area of destruction of the internal elastic lamina. Haematoxylin, alcian green, elastic, Van Gieson $(H A E V G) \times 1000$.

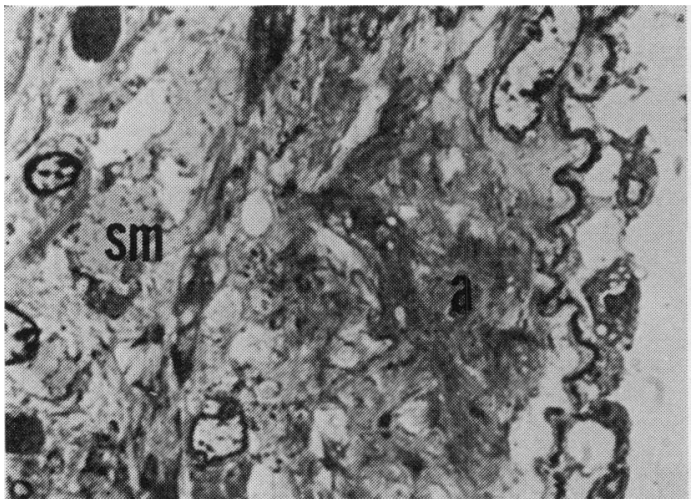

Fig. 5 Plastic-embedded section through an area of very early medial necrosis in an infant of 31 weeks' gestation, 2 days' postnatal age. Media show an acellular area ' $a$ ' where the smooth muscle cells are destroyed and replaced by an amorphous fibrillary mass. Note also vacuolated smooth muscle cells 'sm' scattered through the wall. Toluidine blue $(T B) \times 2200$.

dissection (Fig. 6) with infiltration of red cells through and among degenerate muscle cells of the media. The extent of muscle necrosis in affected vessels varied considerably, from large geographical defects in the media to exquisitely selective focal loss of muscle cells (Figs 7 and 8). A striking feature was the absence of any significant inflammatory reaction and the necrotic lesions were not due to a primary arteritis.

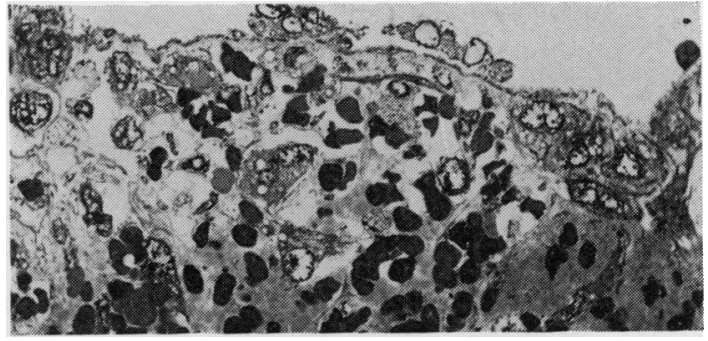

Fig. 6 Plastic-embedded section from the same vessel as in Fig. 5. Luminal surface is in upper segment of figure and several areas of denudation of the intimal cells are present. Striking feature however is presence of large numbers of darkly-stained red cells present within wall of the artery. These red cells dissect between and separate small groups of smooth muscle cells. In this section no break in the internal elastic lamina was noted. TB $\times 1200$.

Accompanying the loss of muscle cells in the media, there was aneurysmal dilatation and microdissection resulting in the formation of false aneurysms. In one infant it was possible to trace the development of a false aneurysm (Fig. 9) through the stages of muscle necrosis, microdissection, and enlargement until it eventually disappeared.

Small, nonocclusive thrombi were quite commonly present in the lumina of damaged vessels (Fig. 10). In these infants the small peripheral vessels within the myocardium contained small thromboemboli 


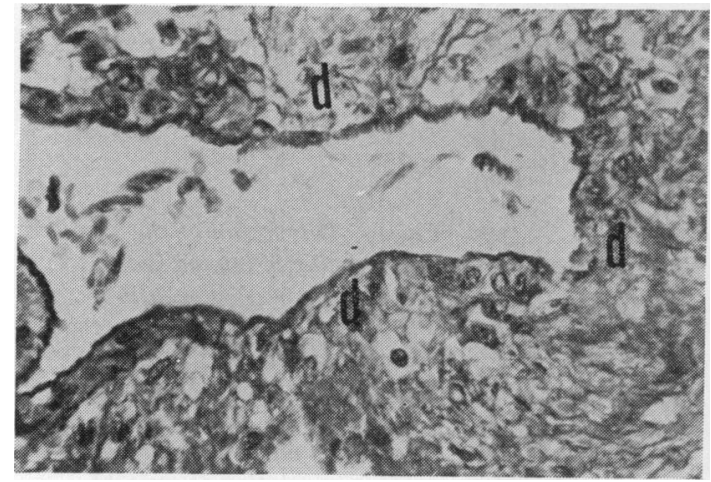

Fig. 7 Section through a branch of the circumflex artery, 35 weeks' gestation, 3-day-old infant, showing large defects ' $d$ ' in the media. Note absence of any inflammatory reaction. HAEVG, $\times 700$.
(Fig. 11), and, occasionally, early organisation of embolus and its incorporation below the endothelium of small vessels could be identified.

The spectrum of changes described in the preceding paragraphs were more commonly seen in the stillbirths and younger infants in the series, and were found more readily in hearts with endocardial and epicardial petechial haemorrhages. The affected vessels varied in size and the largest ones, in this particular series, were the first major branches of both right and left coronary arteries(anterior descending branch of left coronary artery, lateral branch of right coronary artery). In this series acute necrotic lesions of the main right and left coronary arteries were not identified.

The older infants in the series showed a somewhat different spectrum of changes. The least obvious lesions consisted of small scars of media, with
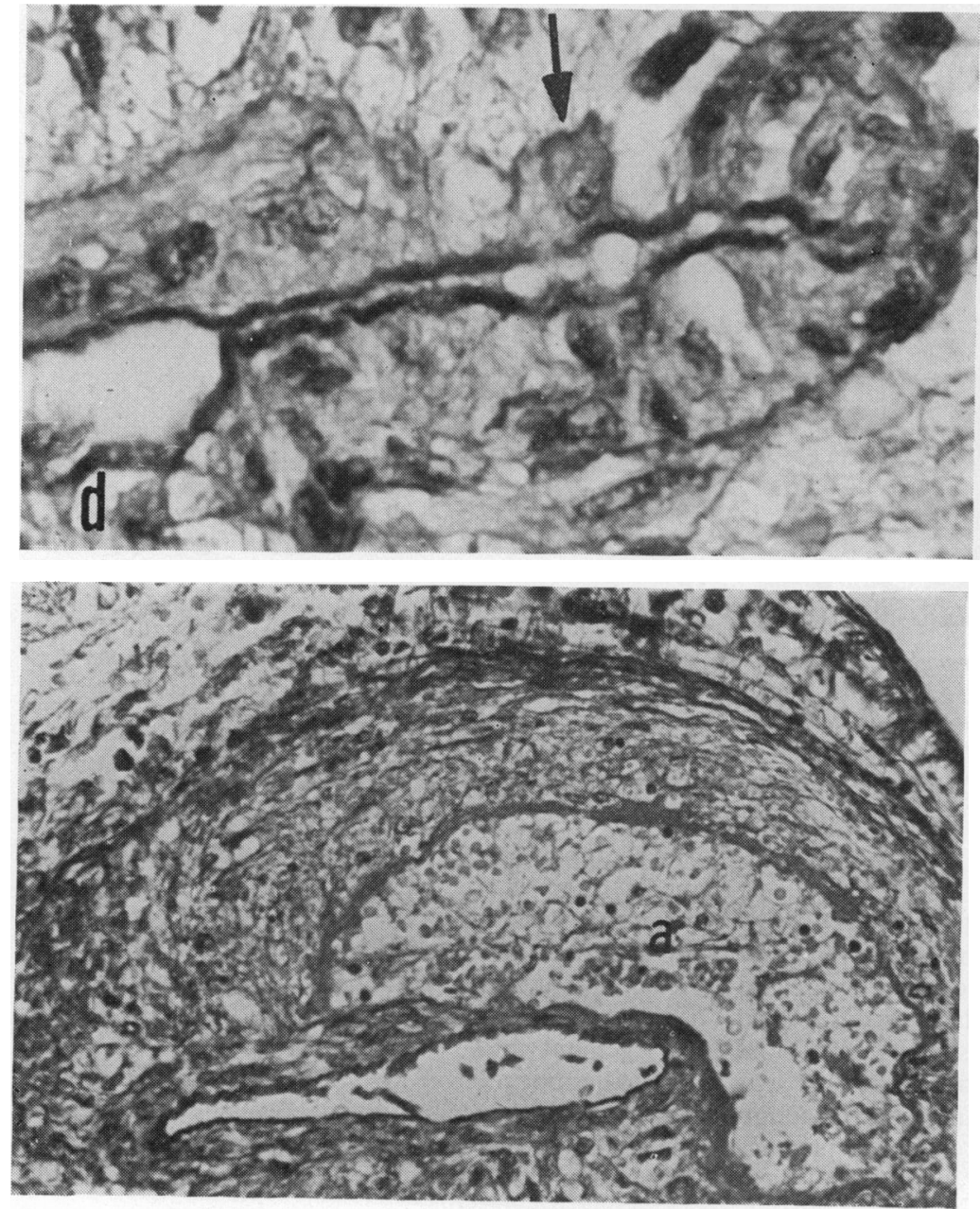

Fig. 8 Same case as Fig. 7 taken from a lateral branch of the anterior descending coronary artery, showing single surviving smooth muscle cell of the media (arrow) and also a larger defect ' $d$ ' in the medial musculature. HAEVG, $\times 1600$.

Fig. 9 Posterior interventricular branch of the right coronary artery in a 39-week-gestation infant aged 9 days. $A$ large false aneurysm of the wall ' $a$ ' is shown. In serial sections its development from areas of microdissection could be traced. $H A E V G, \times 450$. 
excessive accumulation of acid mucopolysaccharide and fragmentation of the elastica (Fig. 12). In many of the older infants different stages in the organisa-

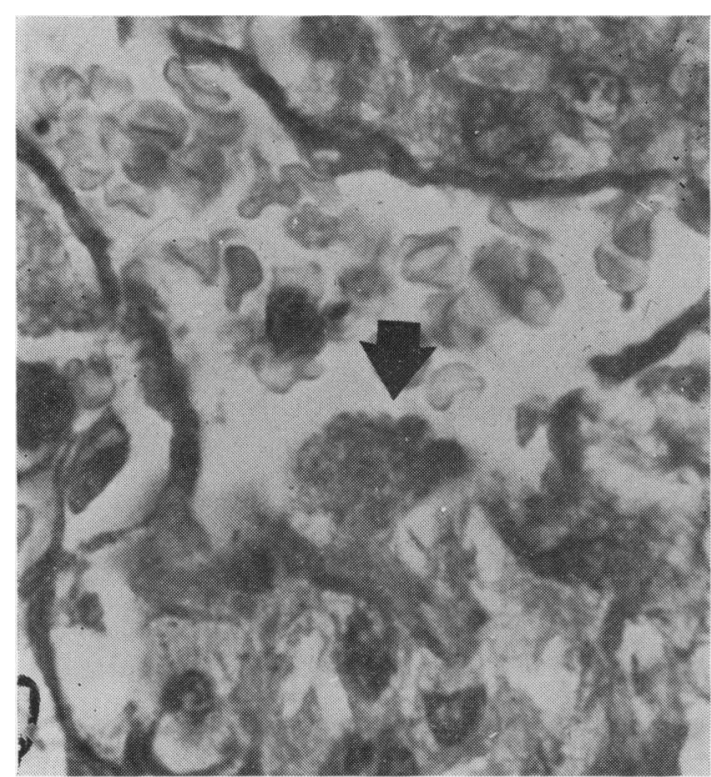

Fig. 10 Serial section from same vessel as Fig. 6 , showing a small mushroom-like thrombus protruding into the vascular lumen (arrow). HAEVG, $\times 4000$.

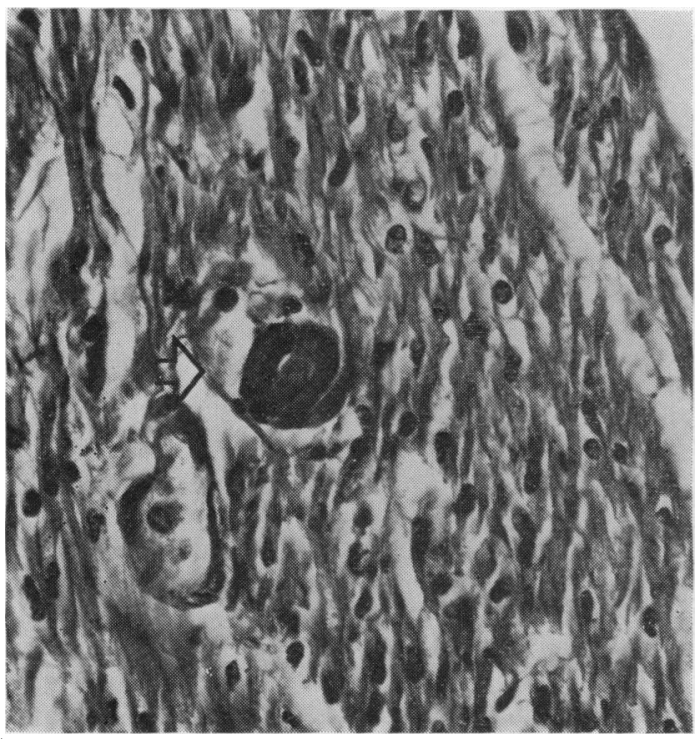

Fig. 11 Intramural thromboembolus (arrow) in 35-week-gestation infant aged 5 days. Embolus is attached to the vessel wall and in other serial sections an early stage of endothelialisation was evident. Martius scarlet blue $(M S B) \times 1000$. tion of thromboemboli within peripheral branches were present, amounting in some instances to obliteration of the lumen (Fig. 13). There was also evidence of progressive recanalisation of the vessel lumen (Fig. 14) and the affected vessels showed very obvious distortion of their architecture (Fig. 15). Many severely damaged vessels, with cellular intimal plaques overlying areas of pronounced medial hypoplasia were present (Fig. 16).

In many of the older infants prominent intimal

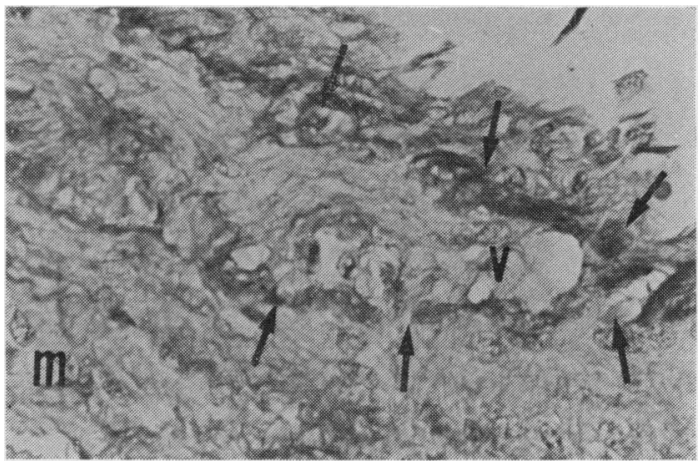

Fig. 12 Circumflex artery in an infant of 26 weeks' gestation, 42 days' postnatal age. There is obvious splitting and reduplication of the internal elastic lamina (arrows). Large vacuoles ' $v$ ' staining faintly with alcian green, indicate presence of abnormal amounts of acid mucopolysaccharides. Note also the defects in the medial elastic tissue ' $m$ '. HAEVG, $\times 700$.

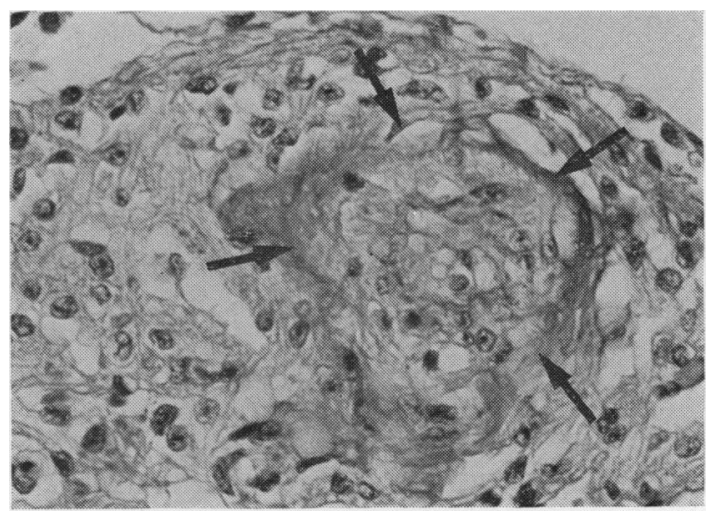

Fig. 13 Section through base of the anterior papillary muscle of the mitral valve in a 3-month-old infant, born at 29 weeks' gestation, who presented as a 'sudden infant death'. An obliterated intramural vessel is outlined by arrows. Serial sectioning traced its origin from an intramural artery shown in Fig. 14. HAEVG, $\times 1000$ 
proliferation was noted in the surface vessels. There was disruption of the internal elastic lamina, irregular smooth muscle proliferation, and excessive deposits of collagen and elastic tissue in the intima (Fig. 17).

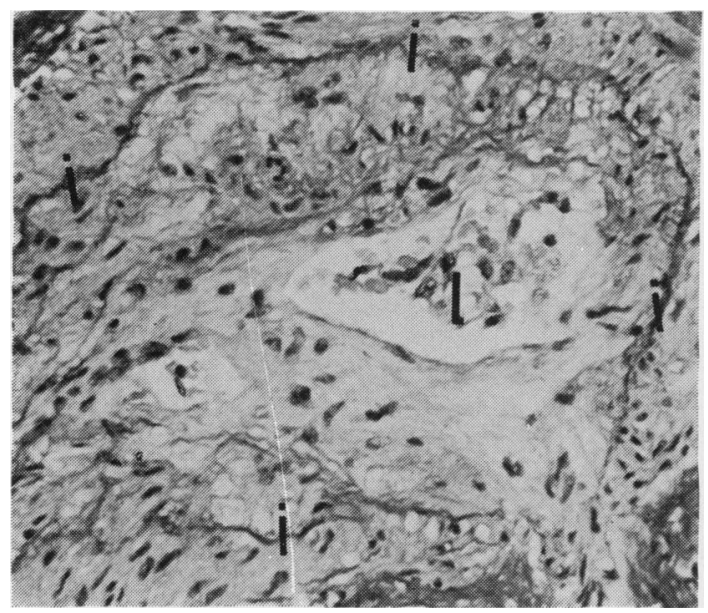

Fig. 14 Same infant as in Fig. 13. Parent vessel of lesion shown in Fig. 13 is seen. There is obvious disorganisation of the architecture. Internal elastic lamina is outlined ' $i$ ' and indicates the approximate size of the original vessel lumen. New vessel lumen ' $L$ ' is considerably narrower. These appearances were interpreted as being due to recanalisation of the vessel after obliteration. $H A E V G, \times 700$.

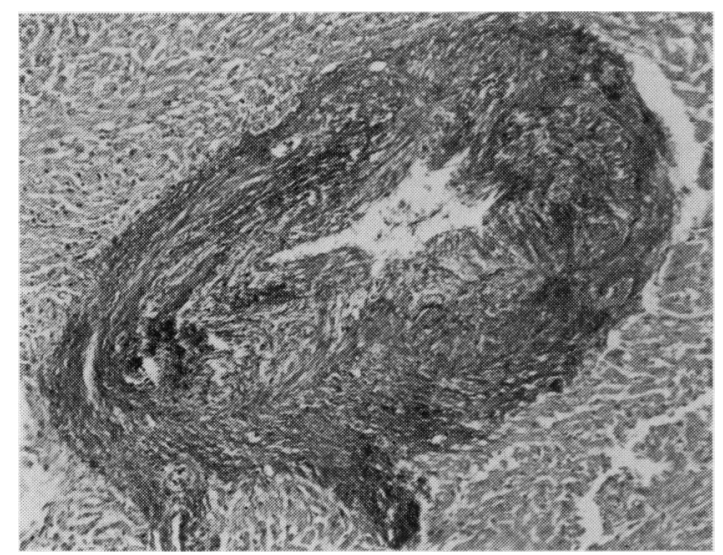

Fig. 15 Complete destruction of architecture is seen in this section from the right ventricle of a 6-month-old infant, born at 39 weeks' gestation with an omphalocele which was repaired, but who required prolonged ventilation. Landmarks of the vessel wall are replaced by darkly-staining bands of elastotic scar tissue. Elsewhere in this section numerous normal vessels were found. $H A E V G, \times 220$.

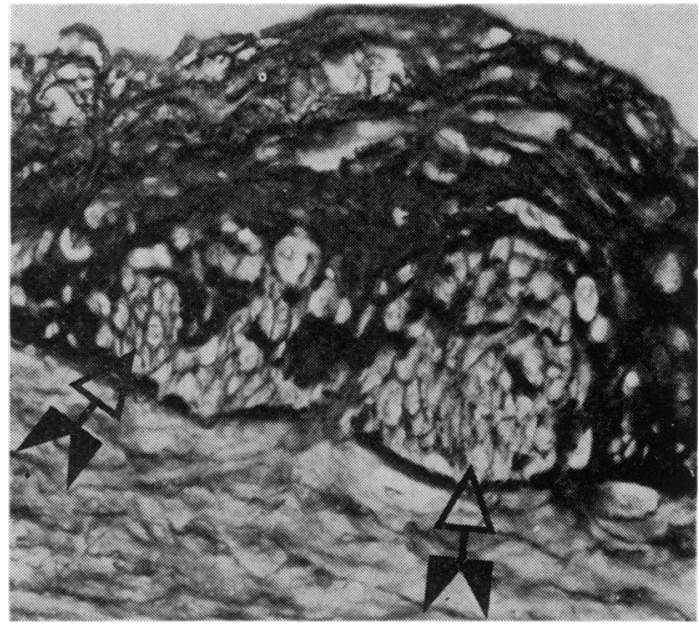

Fig. 17 Small intimal plaque in anterior descending branch of coronary artery in 4-month-old 'sudden infant death'. Smooth muscle nodules (arrows) are present just above the internal elastic lamina, which shows up as an irregularly reduplicated layer. Aldehyde-fuchsine, $\times 1000$. 


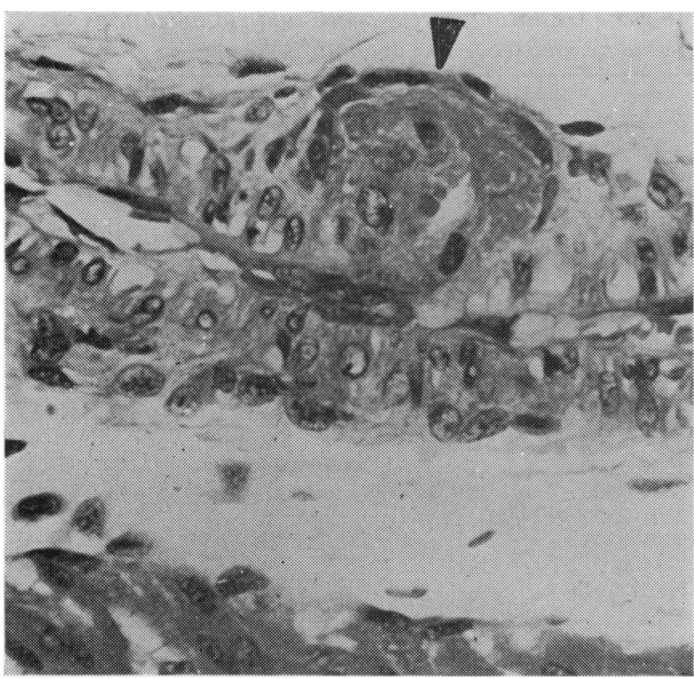

Fig. 18 Intramural myocardial vessel from 2-month-old infant born at 40 weeks' gestation with severe birth asphyxia and with evidence of brain damage. A globular lesion (arrow) filled with fibrillary material, that stained positively for fibrin (not shown), is present. Media at this point of the vessel appear irregular and much less cellular than on the opposite side of the vessel wall section. Lesion was interpreted as a healed false aneurysm. Haematoxylin and eosin $(H \& E), \times 1000$.

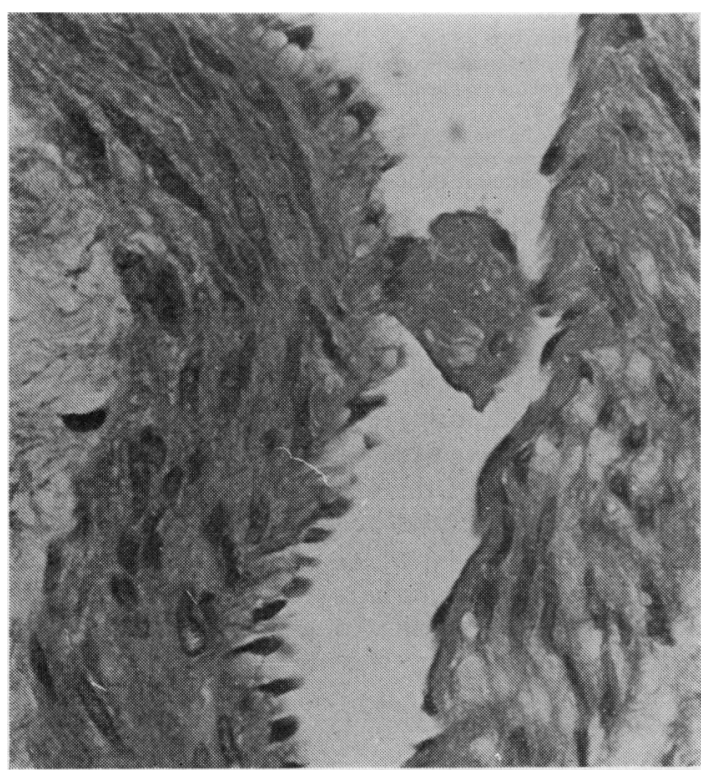

Fig. 19 Organised, pedunculated, thrombus in an infant aged 32 days, born at 26 weeks' gestation. Lesion was seen in a branch of the circumflex artery. $H \& E, \times 1000$.
In some of the older infants occasional zones of acute medial necrosis in both surface and intramural vessels could still be identified. This finding gave substance to the speculation that the degenerative changes seen in the older infants represented the end results of acute lesions seen in younger ones.

Some miscellaneous lesions were also identified. In one infant a rounded globular lesion contained fibrinous material, and was interpreted to be a healed false aneurysm (Fig. 18). Polypoidal structures in some larger vessels almost certainly represented organised thrombi (Fig. 19). Many of the smaller intramural vessels showed the presence of prominent foamy endothelial cells projecting into the lumen, and these were interpreted as representing a reaction to previous emboli.

\section{Ultrastructural studies}

In certain, selected cases, the $1 \mu \mathrm{m}$ thick sections allowed the identification of zones of vacuolated smooth muscle cells in the walls of vessels which were severely damaged (Fig. 20) and representative samples from these areas were taken for ultrastructural studies. The cells themselves contained abundant lipid vacuoles, closely associated with

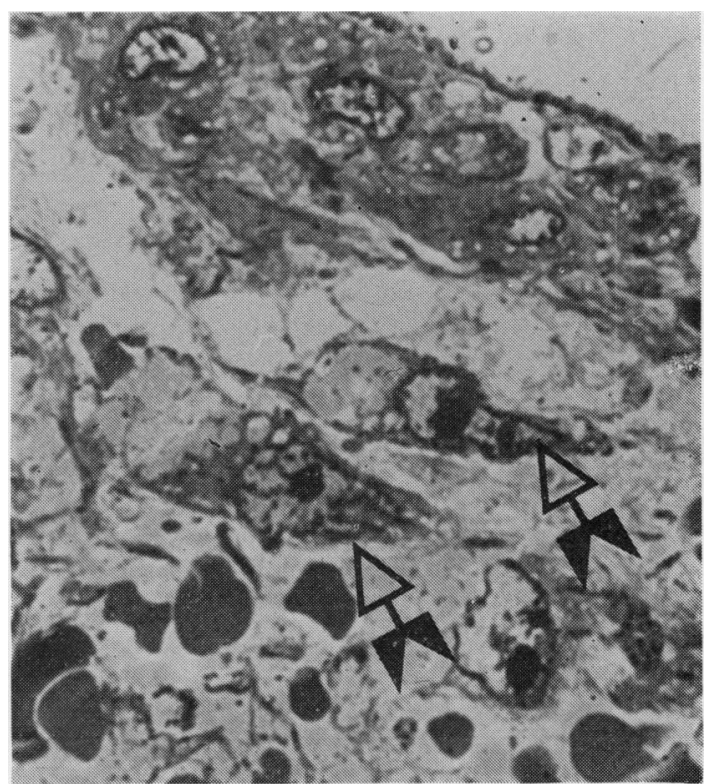

Fig. 20 Section through an epicardial petechial haemorrhage from a stillborn infant of 42 weeks' gestation after a pregnancy complicated by abruptio placenta. Outer layers of media show the presence of grossly distorted smooth muscle cells (arrows), with numerous lipid vacuoles in the cytoplasm. Plastic embedded section, TB $\times 4000$. 
granular endoplasmic reticulum, and many of the lipid vacuoles appeared to contain complex convoluted inclusions resembling myelin figures (Fig. 21). It was thought that these changes represent very early evidence of smooth muscle cell damage.

Associated clinical features. The frequency with which destructive lesions of the coronary arterial tree were found is shown in Table 2, and the high incidence in stillbirths, early neonatal deaths, infants maintained on prolonged ventilation, and sudden infant deaths is obvious. In the whole series nearly one-third of the infants had evidence of coronary arterial lesions.

The complications of pregnancy and postnatal life in the younger infants in this series are shown in Table 3. It can be appreciated that infants with necrotic and degenerative lesions of their coronary vessels were often associated with conditions that could lead to hypoxia both in utero and in postnatal life. In particular antepartum haemorrhage, fetal bradycardia, postnatal apnoeic attacks, respiratory distress syndrome, intraventricular haemorrhage, and any indication for prolonged ventilation were important associations.

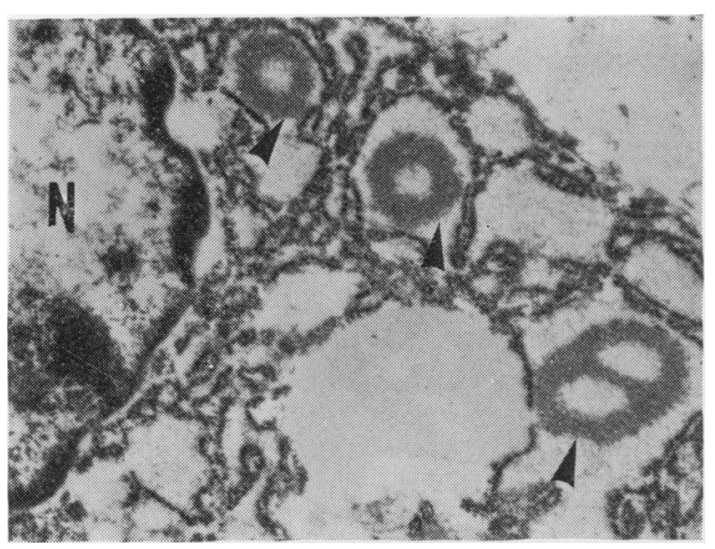

Fig. 21 Electron micrograph of the foamy smooth muscle cells seen in Fig. 20, showing the numerous myelin-like figures in lipid vacuoles in the cytoplasm. $N=$ nucleus. Uranyl acetate and lead citrate; $\times 20000$.

Table 2 Incidence of coronary arterial lesions

\begin{tabular}{lll}
\hline & $\begin{array}{l}\text { Total } \\
(n=256)\end{array}$ & $\begin{array}{l}\text { Affected } \\
(n=79)\end{array}$ \\
\cline { 2 - 3 } & & No. $(\%)$ \\
\hline Fresh stillbirths & 82 & $20(24)$ \\
First week neonatal deaths & 86 & $24(28)$ \\
Infants on prolonged ventilation & 24 & $22(92)$ \\
Other neonatal deaths & 33 & $5(15)$ \\
Sudden infant death syndrome & 13 & $5(38)$ \\
Other infants & 18 & $3(17)$ \\
\hline
\end{tabular}

Table 3 Gestation, birthweight, and complications of pregnancy, delivery, and neonatal life

\begin{tabular}{|c|c|c|}
\hline & $\begin{array}{l}\text { Infants with } \\
\text { arterial } \\
\text { lesions } \\
(n=79)\end{array}$ & $\begin{array}{l}\text { Infants with- } \\
\text { out arterial } \\
\text { lesions } \\
(n=177)\end{array}$ \\
\hline \multicolumn{3}{|l|}{ Gestation (weeks) } \\
\hline 28 or less & 18 & 59 \\
\hline $29-37$ & 37 & 77 \\
\hline $38-41$ & 16 & 23 \\
\hline$>41$ & 2 & 6 \\
\hline Not known & 6 & 12 \\
\hline \multicolumn{3}{|l|}{ Birthweight (g) } \\
\hline$<1000 \mathrm{~g}$ & 16 & 29 \\
\hline $1001-1500$ & 16 & 21 \\
\hline $1501-2000$ & 13 & 46 \\
\hline $2001-2500$ & 10 & 31 \\
\hline $2501-3000$ & 8 & 19 \\
\hline $3001-3500$ & 9 & 16 \\
\hline$>3500$ & 4 & 6 \\
\hline Not known & 3 & 9 \\
\hline \multicolumn{3}{|l|}{ Mode of delivery } \\
\hline Vertex & 36 & 86 \\
\hline Breech & 19 & 37 \\
\hline Caesarean & 12 & 22 \\
\hline Other & 一 & 4 \\
\hline Not known & 12 & 28 \\
\hline \multicolumn{3}{|l|}{$\begin{array}{l}\text { Complications of pregnancy, delivery, } \\
\text { or neonatal life.* }\end{array}$} \\
\hline Antepartum haemorrhage & 32 & $36(P<0.01)$ \\
\hline Maternal hypertension & 14 & 29 \\
\hline Maternal diabetes mellitus & 4 & 3 \\
\hline Prolonged rupture of membranes & 16 & 38 \\
\hline Fetal bradycardia & 58 & $69(P<0.001)$ \\
\hline Meconium staining of liquor & 28 & 62 \\
\hline Apgar score $<6$ at $1 \mathrm{~min} / 5 \mathrm{~min}$ & 56 & 85 \\
\hline Apnoeic attacks & 44 & $56(P<0.001)$ \\
\hline Respiratory distress & 36 & $49(\mathrm{P}<0.01)$ \\
\hline Interstitial emphysema/pneumothorax & 21 & 29 \\
\hline Bronchopulmonary dysplasia & 41 & $48(P<0.001)$ \\
\hline Pneumonia & 6 & 14 \\
\hline Intraventricular haemorrhage & 41 & $68(\mathrm{P}<0.05)$ \\
\hline
\end{tabular}

* Most infants appear more than once.

In view of this apparent association with hypoxia, the clinical histories of the older infants (not maintained on prolonged ventilation) and the 'cot deaths' were reappraised. In particular, evidence of hypoxic episodes or conditions predisposing to hypoxia were searched for. These were present in 5 of the 13 'cot deaths' and in 6 of the 18 older infants (Table 4). Four of the 13 'cot deaths' were stated to have weighed $2500 \mathrm{~g}$ or less at birth. In 3 infants this was associated with maternal hypertension. In the remaining low birthweight infant no other associated anomaly of pregnancy was described. In the fifth 'cot death' there was a history of vaginal bleeding 3 days before delivery. In the 6 older infants, with anomalies of pregnancy and postnatal life, the clinical records were even less detailed than in the 'cot deaths'. Three were stated to have had apnoea at birth; 2 infants were said to have been 'premature' but no further details were given. One infant was 
Table 4 Evidence of perinatal hypoxia (sudden infant deaths and older infants)

\begin{tabular}{|c|c|c|}
\hline Infants & Sudden deaths & Older infants \\
\hline $\begin{array}{l}\text { With coronary } \\
\text { arterial lesions } \\
\text { Without lessions }\end{array}$ & $\begin{array}{l}3 \text { of } 5 \\
2 \text { of } 8\end{array}$ & $\begin{array}{l}2 \text { of } 3 \\
4 \text { of } 15\end{array}$ \\
\hline
\end{tabular}

Table 5 Comparison of coronary, renal, and adrenal arterial lesions

\begin{tabular}{lll}
\hline Infants $(n=256)$ & \multicolumn{2}{l}{ Infants with arterial lesions } \\
\cline { 2 - 3 } & Renal & Adrenal \\
\hline $\begin{array}{l}\text { With coronary arterial lesions } \\
(\mathrm{n}=79) \\
\begin{array}{l}\text { Without coronary arterial lesions } \\
(\mathrm{n}=177)\end{array}\end{array}$ & 6 & 8 \\
\hline
\end{tabular}

delivered by the breech with severe birth asphyxia, and also showed evidence of thalamic and brain stem neuronal loss at necropsy (at age 3 months). She was known to have had severe cerebral damage during life.

\section{Findings in other vessels}

Surprisingly, necrotic and proliferative changes of the variety described in the coronary arterial tree were not seen as commonly in the renal or adrenal vasculature (Table 5). When present in the microvasculature in these areas, they were almost invariably associated with thromboemboli associated with 'high placement' of an umbilical arterial catheter within the aorta, and several of these infants have been described elsewhere (Tyson et al., 1976). In 5 infants the thromboemboli were probably derived from other sources: in 3 infants, fibrinous vegetations were present on both tricuspid and mitral valves. In the other 2 infants, thrombi were noted in pulmonary venules in association with bacterial pneumonitis.

\section{Discussion}

It has been known for a long time that the adequacy of the fetus' and infant's response to asphyxia to a great extent depends on the integrity of the cardiovascular system (Cross, 1966). If the cardiovascular system is damaged the fetus is compromised. In recent years interest in the myocardial function and reserve in perinatal hypoxia or asphyxia has been growing, and there is abundant evidence that in many instances the myocardium is affected in states of severe hypoxia (Burnard and James, 1961; Rowe and Hoffman, 1972; Rowe et al., 1978). While this may reflect direct myocardial damage in hypoxia (de Sa, 1977), the possibility that damage to the coronary arterial tree may be an important factor does'not appear to have been considered, and the earlier observations of Gruenwald (1949) have passed unnoticed. Yet it is clear, that in cases where there is frank myocardial necrosis (Bucciarelli et al., 1977; de Sa, 1977) vascular lesions should be considered and sought for.

The data presented in this report show that coronary arterial lesions, albeit of a focal nature, are common in newborn infants, and that they are associated with myocardial necrosis. Owing to their focal nature, the arterial changes are not always easy to find and can be missed.

It is unlikely that the acute arterial lesions, described in this series of infants, represent the effects of resuscitation measures such as external cardiac massage. Not all our infants (with acute necrotic lesions) were resuscitated by this manner. While it is true that acute lesions were often more readily identifiable in surface vessels, many intramural vessels had similar changes. If it is suggested that the deep intramural vessels were damaged by external trauma, the insult must have been capable of producing exquisitely selective loss in the media of many vessels of varying calibre, while sparing many larger surface vessels and avoiding gross contusions of the myocardium. It is difficult to imagine such a model.

A far more convincing case can be made for the view that the many different arterial lesions seen in this small series of infants form part of a closely related spectrum. The sequence of events would include damage to the media, and in particularly severe cases to the intima, with the development of nonocclusive thrombi. Emboli could lodge in distal intramural vessels with varying degrees of myocardial damage. Repair of the initial vascular damage could explain the development of irregular medial scarring, with or without aneurysmal formation, and the striking intimal lesions seen in the intramural vessels could be traced to the organisation of thromboemboli. Such a sequence of events is known to occur in the vessels of other organs of humans (Salyer et al., 1974; Sevitt, 1976; Tyson et al., 1976), and animals (Allison et al., 1967).

If the sequence of events outlined above is a correct interpretation, some of the earlier statements regarding 'remodelling' of the coronary arterial tree during growth of the fetus and infant need to be reassessed (Robertson, 1960; Ungar, 1965). During this study, numerous intramyocardial arteries were identified and examined closely. Most of them were perfectly 'normal' histologically without the evolution of longitudinal muscle bundles and other changes described by Ungar (1965). In the present series where deviations from the textbook 'normal' were identified, they were seen to be focal in nature and were often in close juxtaposition with 'normal' 
vessels of comparable calibre. As shown in Table 1 they were also often associated with myocardial lesions. One is tempted to agree with Wilens (1951) that most, if not all, intimal changes in any artery are due to pathological processes, and intimal changes in 'remodelled' vessels may be viewed in a similar fashion.

In this series there does not appear to be any relationship between the presence of coronary arterial lesions and a particular period of gestation or range of birthweight. It is easier to relate the lesions to the complications of pregnancy and postnatal life, and in particular to the likelihood of hypoxia or asphyxia that appears to represent the one factor common to them all.

It is well known that in infants dying from intrapartum asphyxia, epicardial and other serosal petechiae are common pathological findings at necropsy (Morison, 1970). The acute coronary arterial lesions presented in this paper were most readily identified in those hearts with epicardial petechiae, as Gruenwald (1949) pointed out earlier. In fact, what is thought to represent the very earliest evidence of arterial wall damage was seen near areas of ruptured, periarterial, epicardial capillaries. It is possible that some of the periarterial capillary vessels function as vasa vasorum. Certainly, retrograde injection of barium/gelatin mixtures into the ascending aorta with filling of the aortic sinuses and main coronary arteries delineate a delicate capillary network around the main coronary vessels and their larger branches (de $\mathrm{Sa}$, unpublished observations). Rupture of these vasa vasorum could then produce the appearance of perivascular, epicardial petechiae. This, in turn, could lead to focal medial necrosis of the arterial wall, which could initiate the postulated sequence of events in the arterial wall.

Morison (1970) suggested that the serosal petechial haemorrhages seen at necropsy, in cases of intrauterine and intrapartum asphyxia associated with antepartum haemorrhage, were related to a great increase in the perfusing pressure in serosal capillaries. It is well known that in other situations an acute, rapid rise in blood pressure (as in the 'malignant' phase of hypertension) may be accompanied by evidence of bleeding due to destruction and rupture of the microvasculature (Goldby and Beilin, 1972). In experimental models, these areas of destruction are associated with fibrinoid necrosis (Allison et al., 1967; Eto et al., 1978). Similar changes are present in human material (Hughson and McManus, 1978). It is also known that in the human infant, one initial response to hypoxia or asphyxia is a sharp rise in blood pressure that may be related to catecholamine release (Dawes, 1968). It is permissible, therefore, to argue that in the asphyxiated baby a sharp rise in blood pressure, occurring as a reaction to the initial insult, may produce the rupture of epicardial vessels (Fig. 22).

For this explanation to be likely, however, it is necessary to explain the relative sparing of the visceral circulation (represented in this paper by the renal and adrenal vessels), for if the coronary vasculature is damaged by an episode of hypertension in response to an acute asphyxial insult, it would appear logical to expect lesions to be present in other viscera as well. The paradox can be explained only if it is postulated further that the splanchnic viscera, such as the adrenals and kidney, are 'protected' from the hypertension by a selective vasospasm. Such an explanation would be analogous to the discrepancy in the state of the arterial tree in the kidneys in cases of hypertension associated with unilateral renal artery stenosis. Here it is known that in the kidney supplied by the stenoticartery thearteries rarely show evidence of fibrinoid necrosis, while the arteries of the 'exposed' contralateral organ are severely affected (Heptinstall, 1966). One would expect that such a 'protective' vasoconstriction, while sparing the arteries, could make the organ prone to ischaemic damage. The association of haemorrhagic necrosis of the adrenal gland in stillbirths and infants with severe hypoxia/asphyxia in utero is well documented (de Sa and Nicholls, 1972), and presumably represents an example of this phenomenon.

It is possible, that if the initial asphyxial episode is severe and acute, and if the pressor response is pronounced there may be a considerable hazard posed to the fetus or newborn infant. It would be possible to construct a vicious cycle with a pressor

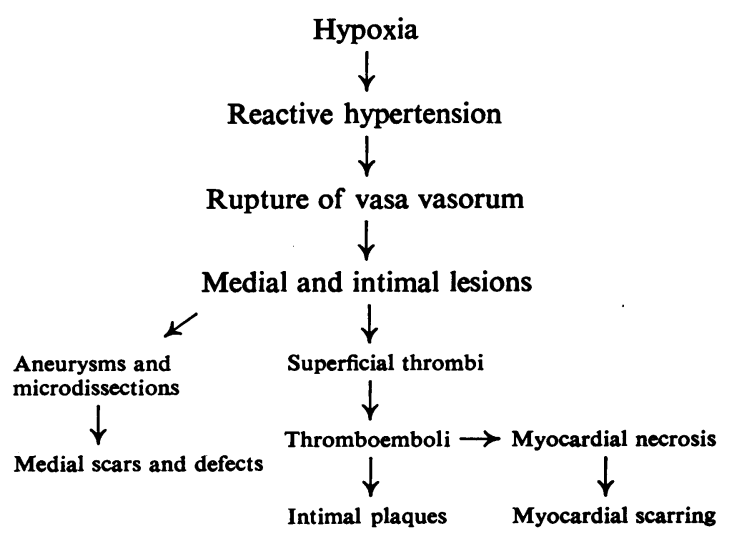

Fig. 22 Suggested scheme for the development of necrotic and degenerative lesions of the coronary arterial tree in infants. 
response to hypoxia or asphyxia damaging the coronary arterial tree and affecting the myocardium secondarily. The damaged myocardium would be unable to respond adequately to the demands placed on it by the original insult, thereby deepening the metabolic complications of the asphyxia. Finally, poor coronary perfusion due to the myocardial damage (de Sa, 1977) would aggravate any original myocardial insult. If such vicious circles exist, extremely complex and severe anomalies of cardiac function could be expected. It was of considerable interest that at least some of the 'cot deaths' had myocardial and coronary arterial lesions strikingly similar to those infants with a well documented history of prolonged hypoxia. In fact, in some of these infants the clinical records suggested that hypoxic episodes had taken place. The findings are consistent, therefore, with some of the more recent views regarding the aetiology of 'cot (crib) deaths' (Naeye, 1973).

It is worth stressing the occurrence of coronary arterial changes in the newborn infant, irrespective of whether or not the pathogenesis of the lesions is known. They represent a serious hazard and may even help to explain how certain unusual lesions of the coronary arterial tree develop. Cases of medial calcification of the coronary vessels (Witzleben, 1970), occlusive fibroelastosis (MacMahon and Dickenson, 1967), coronary sclerosis (Jokl and Greenstein, 1944), congenital aneurysms (Gore et al., 1959), and primary dissecting aneurysms of the coronary vessels in young adults (Whitehead and Dunnill, 1969), could all have their origin in the lesions described in this report. In most of these reported cases, it is difficult to trace any direct connection with anomalies of the antenatal and postnatal period, since details on these points are often scarce, not available, or not even considered. This is not entirely surprising, but in any future studies of coronary vessels in infants and children, it would be useful to ascertain as much information as possible regarding the antenatal and postnantal periods of development.

Finally, while it is clear that the lesions of the coronary artery can heal, it is equally likely that once damaged no vessel will remain perfectly normal, and it is tempting to suggest that small scars of the coronary arterial tree could predispose to the more severe plaques of later life.

I am grateful to many colleagues in the Department of Pathology and the Hamilton District Program in Laboratory Medicine for access to their material and other help, and to Sharryn Putns, Marion Leskovec, Stephanie Whittaker, and George Turcon for excellent technical assistance.

\section{References}

Allison, P. R., Bleehan, N., Brown, W., Pickering, G. W., Robb-Smith, A. H. T., and Russell, R. P. (1967). The production and resolution of hypertensive vascular lesions in the rabbit. Clinical Science, 33, 39-51.

Berry, C. L. (1967). Myocardial ischemia in infancy and childhood. Journal of Clinical Pathology, 20, 38-41.

Bucciarelli, R. L., Nelson, R. M., Egan, E. A., II, Eitzman, D. V., and Gessner, I. H. (1977). Transient tricuspid insufficiency of the newborn: a form of myocardial dysfunction in stressed newborns. Pediatrics, 59, 330-337.

Burnard, E. D., and James, L. S. (1961). Failure of the heart after undue asphyxia at birth. Pediatrics, 28, 545-565.

Cross, K. W. (1966). Resuscitation of the asphyxiated infant. British Medical Bulletin, 22, 73-78.

Dawes, G. S. (1968). Foetal and Neonatal Physiology, pp. 181-185. Year Book Medical Publishers: Chicago.

de Sa, D. J. (1977). Myocardial changes in immature infants requiring prolonged ventilation. Archives of Disease in Childhood, 52, 138-147.

de Sa, D. J., and Nicholls, S. (1972). Haemorrhagic necrosis of the adrenal gland in perinatal infants: a clinicopathological study. Journal of Pathology, 106, 133-149.

Dock, W. (1946). The predilection of atherosclerosis for the coronary arteries. Journal of the American Medical Association, 131, 875-878.

Esterly, J. H., and Oppenheimer, E. H. (1967). Some aspects of cardiac pathology in infancy and childhood. IV. Myocardial and coronary lesions in cardiac malformations. Pediatrics, 39, 896-903.

Eto, T., Onoyama, K., Tanaka, K., Omae, T., and Torao, Y. (1978). Early vascular changes in the intestine of bilaterally nephrectomised rats. Journal of Pathology, 124, 141-148.

Fangman, R. A., and Hellwig, C. A. (1947). Histology of coronary arteries in newborn infants. American Journal of Pathology, 23, 901-902.

Franciosi, R. A., and Blanc, W. A. (1968). Myocardial infarcts in infants and children. I. A necropsy study in congenital heart disease. Jouinal of Pediatrics, 73, 309-319.

Gault, M. H., and Usher, R. (1960). Coronary thrombosis with myocardial infarction in a newborn infant. New England Journal of Medicine, 263, 379-382.

Goldby, F. S., and Beilin, L. J. (1972). How an acute rise in arterial pressure damages arterioles. Electron microscopic changes during angiotensin infusion. Cardiovascular Research, 6, 569-584.

Gore, I., Smith, J., and Clancy, R. (1959). Congenital aneurysms of the coronary arteries with report of a case. Circulation, 29, 221-227.

Gruenwald, P. (1949). Necrosis in the coronary arteries of newborn infants. American Heart Journal, 38, 889-897.

Heptinstall, R. H. (1966). Pathology of the Kidney, pp. 157-158. Little, Brown: Boston.

Hughson, M. D., and McManus, J. F. A. (1978). Cellular arteriolar nodules in the heart and pancreas in patients with treated malignant hypertension (abstract). Laboratory Investigation, 38, 349.

Jokl, E., and Greenstein, J. (1944). Fatal coronary sclerosis in a boy of ten years. Lancet, 2,659 .

Levene, C. I. (1956). The early lesions of the atheroma in the coronary arteries. Journal of Pathology and Bacteriology, 72, 79-82.

Lober, P. H. (1953). Pathogenesis of coronary sclerosis. Archives of Pathology, 55, 357-383.

MacMahon, H. E., and Dickenson, P. C. T. (1967). Occlusive fibroelastosis of the coronary arteries in the newborn. Circulation, 35, 3-9. 
Minkowski, W. L. (1947). The coronary arteries of infants. American Journal of Medical Sciences, 214, 623-629.

Moon, H. D. (1957). Coronary arteries in fetuses, infants, and juveniles. Circulation, 16, 263-267.

Morison, J. E. (1970). Foetal and Neonatal Pathology, third edition, pp. 169-170. Butterworth: London.

Naeye, R. L. (1973). Pulmonary arterial abnormalities in the sudden-infant-death syndrome. New England Journal of Medicine, 289, 1167-1170.

Robertson, J. H. (1960). Stress zones in foetal arteries. Journal of Clinical Pathology, 13, 133-139.

Rowe, R. D., and Hoffman, T. (1972). Transient myocardial ischemia of the newborn infant: a form of severe cardiorespiratory distress in full-term infants. Journal of Pediatrics, 81, 243-250.

Rowe, R. D., Izukawa, T., Mulholland, H. C., Bloom, K. R., Cook, D. H., and Swyer, P. R. (1978). Nonstructural heart disease in the newborn. Observations during one year in a perinatal service. Archives of Disease in Childhood, 53, 726-730.

Salyer, W. R., Salyer, D. C., and Hutchins, G. M. (1974). Local arterial wall injury caused by thromboemboli. American Journal of Pathology, 75, 285-300.

Schornagel, H. E. (1956). Intimal thickening in the coronary arteries of infants. Archives of Pathology, 62, 427-432.

Sevitt, S. (1976). Arterial wall lesions after pulmonary embolism, especially ruptures and aneurysms. Journal of Clinical Pathology, 29, 665-674.

Tyson, J. E., de Sa, D. J., and Moore, S. (1976). Thromboatheromatous complications of umbilical arterial catheterisation in the newborn: clinicopathologic study. Archives of Disease in Childhood, 51, 744-754.

Ungar, H. (1965). Intramural branches of the coronary arteries (abstract). Lancet, 2, 1175.

Vlodaver, Z., Abramovici, A., Neufeld, H. N., and Liban, E. (1967). Coronary arteries in Yemenites. Journal of Atherosclerosis Research, 7, 161-170.

Whitehead, R., and Dunnill, M. S. (1969). Primary dissecting aneurysms of coronary arteries. Journal of Pathology, 99, 33-37.

Wilens, S. L. (1951). The nature of diffuse intimal thickening of arteries. American Journal of Pathology, 27, 825-839.

Witzleben, C. L. (1970). Idiopathic infantile arterial calcification-a misnomer? American Journal of Cardiology, 26, 305-309.

Correspondence to Dr D. J. de Sa, Department of Pathology, McMaster University, 1200 Main Street West, Hamilton, Ontario L8S 4J9, Canada.

Received 19 February 1978 